\title{
Herpès palatin récurrent
}

\author{
Semaan Abi Najm, Marc El Hage, Jacky Samson* \\ École de Médecine dentaire, Division de Stomatologie et Chirurgie orale, Rue Barthélemy-Menn 19, 1211 Genève 4, Suisse \\ *jacky.samson@unige.ch
}

(Reçu le 11 janvier 2012, accepté le 13 février 2012)

Mots clés :

HSV1 / herpès récurrent / herpès labial / herpès palatin

Key words:

HSV1 / recurrent herpes /

labial herpes / palatal herpes
Résumé - L'herpés labial récurrent, communément appelé bouton de fièvre ou feux sauvages par nos confrères nord-américains, est dû à l'herpes simplex virus de type 1 (HSV1). Il correspond à une réactivation de virus persistant à l'état latent dans le ganglion de Gasser après la primo-infection; cette réactivation, favorisée par différents facteurs, entraine une migration centrifuge du virus qui donne généralement une lésion labiale mais qui peut aussi aboutir à une excrétion asymptomatique de virus.

Ce schéma classique ne correspond pas totalement à la réalité. Quelquefois, l'herpès récidivant peut intéresser la muqueuse buccale, en particulier celle la fibro-muqueuse palatine. Il est important de connaître ces formes car le diagnostic de l'herpès récidivant reste avant tout clinique.

Abstract - Palate recurrent herpes. Recurrent labial herpes, also known as cold sore, night fever, fever blister and wild fires by our North American colleagues, is due to herpes simplex virus type 1 (HSV1). It corresponds to a reactivation of latent virus in the Gasser ganglion after the primo-infection. Many factors contribute to this reactivation which leads to a centrifugal viral migration: this gives generally a labial lesion but also an asymptomatic viral excretion.

This classic schema does not completely correspond to the reality. Sometimes recurrent herpes can concern the oral mucosa, in particular the one of the palate. It is very important to know these forms since the diagnosis of a recurrent herpes is mainly clinical.

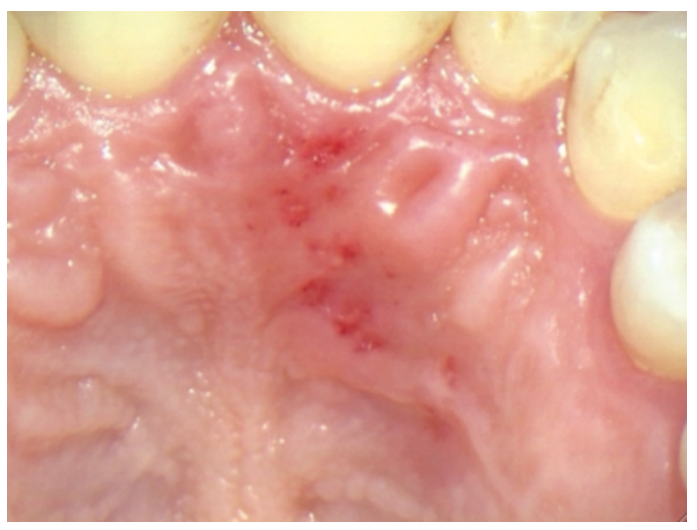

Fig. 1. Aspect de la lésion palatine lors de la consultation en janvier 2012 : ulcérations superficielles, à disposition linéaire, sans érythème associé.

Fig. 1. Appearance of the palatal lesion on january 2012: superficial ulcerations in a linear arrangement without erythema.

Une patiente âgée de 55 ans est venue consulter en janvier 2012 pour une lésion douloureuse palatine antérieure, évoluant depuis 24 heures. Dans les antécédents, on note un cancer du sein à l'âge de
41 ans avec développement de métastases 12 ans plus tard, traitées par chimiothérapie. Depuis 6 ans, elle a présenté plusieurs épisodes identiques, avec une fréquence de 1 à 2 par an. Chaque épisode s'est traduit par une lésion unique douloureuse, siégeant tantôt sur le palais droit, tantôt sur le palais gauche, à hauteur de la région prémolaire-molaire. Lors du dernier épisode, 24 heures après l'apparition de la lésion, on observait des ulcérations superficielles, punctiformes, àdisposition linéaire antéro-postérieure, venant presque au contact du bord libre de la gencive, en arrière de la 21 ; on notait l'absence d'érythème (Fig. 1). Comme pour les épisodes précédents, les douleurs étaient vives, puis elles se sont estompées en 48 à 72 heures et la lésion a disparu en 5 jours. Cette patiente est venue consulter car, en raison de la localisation antérieure de la lésion, elle n'était pas convaincu qu'il s'agissait d'un nouvel épisode d'herpès récidivant car les lésions avaient habituellement un siège plus postérieur (Fig. 2). 


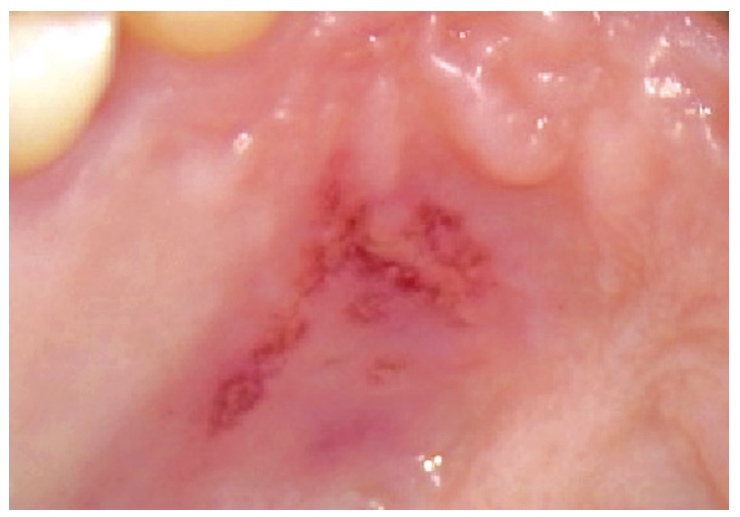

Fig. 2. Aspect de la première lésion palatine, 6 ans auparavant : ulcérations superficielles qui ont tendance à confluer et qui siègent au centre d'une plage érythémateuse.

Fig. 2. Appearence of the first palatal lesion, 6 years ago: superficial ulcerations which are localized in the middle of an erythematous area and trend to join together.

Les herpes simplex virus comportent 2 types (HSV1 et HSV2). Classiquement, HSV1 infecte la partie supérieure du corps, HSV2 la région génito-anale et il est responsable d'une infection sexuellement transmissible et de la contamination éventuelle du nouveau-né lors du passage de la filière génitale. Cette distinction n'a plus de caractère absolu : en raison de l'évolution des pratiques sexuelles oro-génitales, on peut retrouver du HSV2 dans des lésions herpétiques buccales (en particulier chez les sujets infectés par le VIH), et $20 \%$ des récidives et $50 \%$ des primo-infections herpétiques génitales sont dues à HSV1; on peut même observer des primo-infections herpétiques bipolaires $[1,2,3]$.

On considère que $80 \%$ des adultes sont porteurs du HSV1 et que seulement $20 \%$ présentent un herpès labial récurrent; ceci relèverait d'une susceptibilité d'origine génétique [3]. Certains facteurs déclenchant les récurrences sont bien connus: l'hyperthermie, l'infection aiguë fébrile, l'exposition aux ultraviolets, l'asthénie, le stress, les lésions tissulaires dans la région oro-faciale. La plupart des récurrences siègent sur la limite cutanéo-muqueuse des lèvres mais quelques récidives intéressent le menton, les orifices narinaires, les joues et la cavité buccale, le plus souvent la fibro-muqueuse palatine. En conséquence, il serait plus pertinent de parler d'herpès oro-facial. La contamination peut se faire lors d'une primoinfection herpétique, d'une récurrence ou d'une excrétion asymptomatique intermittente. Cette dernière a surtout été bien étudiée dans l'herpès génital : elle est présente entre
2 à $8 \%$ du temps lorsqu'on la détecte par culture mais jusqu'à $28 \%$ lorsqu'on la détecte par PCR. Elle constituerait le tiers de l'excrétion virale, ce qui la fait apparaitre comme le principal mode de contamination. Sur les lèvres, dans la cavité buccale et dans la salive, on peut retrouver du HSV1 en absence de lésions en évolution mais on n'a guère de précision sur la durée de l'excrétion asymptomatique $[1,2,4]$.

Dans la cavité buccale, l'herpès récurrent intéresse presque toujours la fibro-muqueuse palatine, à hauteur des premières molaires, et les lésions successives se développent le plus souvent dans la même région. Les prodromes caractéristiques de toute récidive herpétique font rapidement place à de vives douleurs. La lésion initiale est constituée de vésicules que l'on n'observe jamais car elles font place à des ulcérations punctiformes, regroupées en bouquet, plus ou moins confluentes (respectivement Figs. 2 et 1). L'anamnèse et l'aspect clinique sont souvent suffisamment caractéristiques pour porter le diagnostic qui est confirmé par l'évolution spontanément favorable en quelques jours chez le sujet immunocompétent $[1,2]$.

En conclusion, cette patiente présentait une infection herpétique récurrente particulière par son siège palatin et sa localisation changeante. De plus, il est inhabituel d'observer des récurrences apparaissant aussi tardivement alors qu'habituellement, elles se rencontrent surtout chez des sujets jeunes et que leur fréquence diminue avec l'âge.

\section{Conflits d'intérêt : aucun}

\section{Références}

1. Collège des enseignants de Dermatologie. Infections à herpès virus de l'enfant et de l'adulte immunocompétents: herpès cutané et muqueux. Ann Dermatol Vénéréol 2008;135(11S):18-24.

2. HAS. Prise en charge de l'herpès cutanéo-muqueux chez le sujet immunocompétent (manifestations oculaires exclues). Décembre 2001. Ann Dermatol Vénéréol 2002;129:469-76.

3. Kriesel JD, Jones BB, Matsunami N, Patel MK, St. Pierre CA, Kurt-Jones EA, Finberg RW, Leppert M, Hobbs MR. C21orf91 genotypes correlate with herpes simplex labialis (cold sore) frequency: description of a cold sore susceptibility gene. $J$ Infect Dis 2011;204:1654-62.

4. Wikipedia.org/wiki/herpes_simplex 